\title{
Impact of Maternal Body Mass Index on the Accuracy of Third Trimester Sonographic Estimation of Fetal Weight
}

Juliana Martins ( $\square$ martinjg@evms.edu )

Eastern Virginia Medical School

Tetsuya Kawakita

EVMS Salaried Faculty - Maternal Fetal Medicine/ OBGYN

Priyanka Jain

University of Virginia Maternal Fetal Medicine Fellow (PGY-5)

Margot Gurganus

University of Virginia OBGYN Resident (PGY-3)

Dana Baraki

EVMS Medical Student - Maternal Fetal Medicine / OBGYN

Carole Barake

UTMB OBGYN Resident (PGY-1)

Elena Sinkovskaya

EVMS Salaried Faculty - Maternal Fetal Medicine / OBGYN

Alfred Abuhamad

EVMS Salaried Faculty - Maternal Fetal Medicine/OBGYN

\section{Research Article}

Keywords: maternal BMI, obesity, ultrasound fetal weight estimation, neonatal birthweight

Posted Date: February 7th, 2022

DOI: https://doi.org/10.21203/rs.3.rs-1325095/v1

License: (c) (i) This work is licensed under a Creative Commons Attribution 4.0 International License.

Read Full License 


\section{Abstract}

Objectives: Determine whether maternal body mass index (BMI) can affect the accuracy of sonographic estimation of fetal weight (EFW) in the third trimester when compared to neonatal birthweight (BW).

Methods: Secondary analysis from our original prospective cohort of pregnant women beyond 34 weeks, distributed in 4 groups according to their BMI: normal, overweight, obese and morbid obese. Fetal biometry and fluid measurements were obtained by two experienced sonographers, blinded for patient's clinical information and to each other's measurements. Average EFW and neonatal BW were converted into gestational specific Z-scores. Interobserver correlation coefficient (ICC) and Cronbach's reliability coefficient (CRC) were calculated. Bland-Altman (BA) plots were constructed to assess the level of accuracy.

Results: 100 women were enrolled (800 measurements obtained by 17 sonographers): 17 had normal BMI (17\%), 27 were overweight (27\%), 29 were obese (29\%) and 27 were morbidly obese (27\%). There was no statistical difference for GA at delivery $(p=0.74)$, EFW $(p=0.05)$ or BW $(p=0.09)$ between groups (Table 1). Mean Z-score for EFW was -0.17 (SD 0.81) and for neonatal BW was -0.25 (SD 0.74). ICC was 0.69 $(95 \% \mathrm{Cl} 0.57,0.78)$ and CRC was 0.82 . Mean Z-score difference was small (Table 2). When stratifying according to $\mathrm{BMI}$ categories, the ICC ranged from 0.49 to 0.76 . Reliability indices ranged from 0.66 to 0.86. The Z-scores differences were overall small with no statistical difference (Table 3). BA showed evenly distributed interobserver differences (Figure 1).

Conclusions: When performed by trained sonographers, fetal weight estimation in the third trimester is accurate when compared to neonatal birthweight at increasing BMI categories.

\section{Introduction}

Estimated fetal weight (EFW) is a representative measure for neonatal weight and as such, is an important predictor for neonatal and infant morbidity and mortality ${ }^{1,2}$. In the third trimester, an accurate assessment of fetal weight has valuable information for planning time and mode of delivery 2 .

The limitation of ultrasound estimation of the fetal weight is that it has inherited inaccuracies related to different formulas used and interobserver variability ${ }^{1}$. Previous studies that evaluated ultrasound estimation of the fetal weight found that the accuracy of EFW is compromised by large intra- and interobserver variability ${ }^{3}$ and that most formulas are relatively accurate at predicting birth weight up to 3,500 grams, with overestimation and underestimation of small and large fetuses, respectively ${ }^{2}$.

The prevalence of obesity has dramatically increased over the past decades and now affects more than one in three women in the United States ${ }^{4,5}$. The impact that maternal obesity has on the ultrasound accuracy for the detection of anomalies has been reported in previous studies ${ }^{6-10}$. Limited data exists with regards to the influence of maternal obesity on the accuracy of ultrasound fetal weight estimation ${ }^{6}$, 
with conflicting results ${ }^{11-16}$. Studies on this subject have been overall limited by lack of stratification into obese categories ${ }^{1,12,13}$, and comparison between estimated fetal weight with neonatal weight ${ }^{12}$. Also, most of the studies evaluated the outcome by determining the absolute percentage error between sonographic estimated fetal weight and actual birthweight ${ }^{6,12,14,15,17}$ instead of using Z-scores which is considered the method of choice to assess accuracy.

The aim of this study was to determine whether maternal BMI can affect the accuracy of sonographic estimation of the fetal weight in the third trimester when compared to neonatal birthweight (BW).

\section{Methods}

This study is a secondary analysis from our prospective observational cohort study ${ }^{18}$ published by the same authors and performed at Eastern Virginia Medical School (EVMS), between May, 2018 to July, $2019^{18}$.

Our original study ${ }^{18}$ included all women with singleton pregnancies at or beyond 34 weeks of gestation, above 18 years old and with a BMI above 18.5. Exclusion criteria were all women with twins or higherorder pregnancies, presence of diabetes (pregestational or gestational) or any conditions that could affect the fetal growth potential such as growth restriction, structural or genetic abnormalities. Lack of reliable dating was also an exclusion criteria.

Women enrolled to the study were placed into 4 groups according to their BMI at the time of recruitment: normal (BMI of 18.5 - 24.9), overweight (BMI of 25 - 29.9), obese (BMI of $30-39.9$ ) and morbidly obese (BMI of 40 or greater). BMI was calculated at the time of recruitment to allow a more precise evaluation of the maternal habitus at the time of the scan.

Fetal biometry and amniotic fluid were obtained independently by two sonographers or physicians who were blinded to any clinical information related to the patient and to each other's measurements. The portion of the ultrasound screen displaying the gestational age was covered from the sonographers to minimize bias.

Biometric measurements included in the study protocol were the biparietal diameter (BPD), head circumference $(\mathrm{HC})$, abdominal circumference $(\mathrm{AC})$ and femur length (FL). Main vertical pocket (MVP) and amniotic fluid (AFI) were obtained for the fluid assessment. All measurements followed recent published criteria ${ }^{19}$. Estimated fetal weights were calculated based on the Hadlock et al 1985 biometric equation ${ }^{20}$. Neonatal birthweights were recorded at delivery.

Proficient sonographers with over 4 years of experience in our ultrasound unit, certified by the Registered Diagnostic Medical Sonographer (RDMS) and physicians that meet the American Institute of Ultrasound in Medicine physician qualifications for the performance of obstetric ultrasound examination (https://www.aium.org/resources/guidelines/obstetric.pdf) completed all the ultrasound exams. Our ultrasound unit has been AIUM accredited for the performance of obstetrical sonography for more than 
15 years. The ultrasound equipment used for the study was the Voluson E6, E8 and E10 (GE Healthcare Ultrasound - Zipf, Austria) with a 4-8 MHz or $6 \mathrm{MHz}$ transabdominal probe.

This study was approved by the Institution's Review Board (IRB\#20-12-EX-0259) and written informed consent was obtained from all study subjects.

The primary outcome of the study was the accuracy of sonographic estimation of fetal weight in the third trimester when compared to neonatal birthweight according to BMI categories.

\section{Statistical Analysis}

Statistical analyses were performed using Stata/IC 16.1 (StataCorp, College Station, TX).

Because there is a time lag between ultrasound evaluation and delivery, we converted an average of EFW by two sonographers or physicians and neonatal birthweights to gestational specific Z-scores. Interobserver correlation (ICC) coefficients and Cronbach's reliability coefficient were calculated between Zscores of EFW and neonatal birthweights. An ICC value of 1.00 was considered perfect accuracy between sonographers, while a value $>0.70$ was considered very high level of accuracy. Cronbach's alpha $>0.70$ was considered to demonstrate good internal consistency. Bland-Altman plots were constructed to assess the level of accuracy according to BMI categories. This allows assessment of systematic bias and $95 \%$ limits of agreement. All hypothesis testing was carried out at the $95 \%$ significance level, unless otherwise specified, with a $p$ value of $<.05$ accepted as statistically significant.

\section{Results}

\section{Demographics}

A total of 110 women were enrolled, resulting in a total of 220 ultrasound studies, 880 fetal biometric measurements obtained by 17 different sonographers or physicians. A total of 10 women were excluded due to lack of neonatal data, resulting in 200 studies. Seventeen (17\%) women had a normal BMI, 27 (27\%) women were overweight, 29 (29\%) women were obese, and 27 (27\%) women were morbidly obese.

Maternal demographics are presented in Table 1. The mean gestational age was 36.8 weeks ( \pm standard deviation 1.5) and mean maternal BMI was 31.3 ( \pm standard deviation 9.0$)$ at the time of ultrasound. There was no statistical difference for gestational age at delivery $(p=0.74)$, EFW by ultrasound $(p=0.05)$ or birth weight $(p=0.09)$ between groups. The first author $(J M)$ performed 80 out of the 200 ultrasounds.

\section{Fetal Estimated weight and Neonatal Birthweight}

Statistics of the estimated fetal weight and neonatal weight are shown in Table 2. The mean Z-score for fetal sonographic estimated weight was -0.17 (SD 0.81) and for neonatal birthweight was -0.25 (SD 0.74). The estimated ICC was $0.69(95 \% \mathrm{Cl} 0.57,0.78)$ and reliability coefficient was 0.82 . The mean Z- 
score difference was small, ranging from -1.14 to 1.31. Z-scores for EFW and neonatal BW are expressed Table 2.

\section{Differences by BMI}

Fetal sonographic estimated weight and neonatal weight and mean Z-score difference according to BMI groups are presented in Table 3. The ICC ranged from 0.49 (overweight group) to 0.76 (morbidly obese group). Reliability indices ranged from 0.66 (overweight group) to 0.86 (morbidly obese group).

The Z-scores differences were overall small, ranging from 0.03 to 0.14 with no statistical difference between groups.

Bland-Altman plots for accuracy between sonographic estimated fetal weight and neonatal birthweight are presented in Figure 1. The interobserver differences were overall evenly distributed above and below the zero-difference line, suggesting no systematic errors between observers. Interobserver differences for the measurements primarily fell within the $95 \%$ limits of agreement.

\section{Discussion}

In this study, we evaluated the accuracy of fetal sonographic weight estimation in the third trimester according to neonatal weight in different BMI categories, with stratification into obese categories ${ }^{1,12,13}$, and with adequate representation of obese / morbidly obese group ${ }^{12-14}$. Also, most of the studies evaluated the outcome by determining the absolute percentage error between sonographic estimated fetal weight and actual birthweight 6,12,14,15,17. Our study used Z-scores as it allows a straight comparison of variation over a range of gestational ages.

Understanding whether maternal obesity decreases the accuracy of ultrasound fetal weight estimation in the third trimester is crucial, as this is the time in pregnancy when fetal growth evaluation tends to influence clinical decisions the most, especially in the setting of abnormally grown fetuses ${ }^{21,22}$. In a large retrospective cohort, ultrasound estimation of fetal weight was an independent risk factor for cesarean delivery, showing that it plays a major influence in delivery management ${ }^{23}$.

We found that when comparing Z-scores differences, the accuracy of fetal sonographic weight estimation did not change based on the different BMI categories. Our findings may have been influenced by the presence of experienced sonographers and physicians, state-of-the-art ultrasound systems and a high volume of increased BMI patients, resulting in increased expertise in the obstetric ultrasound in pregnancies with high BMI.

The ICC and reliability coefficient paradoxically increased in the obese and morbidly obese (highest values) category as BMI increased, though this was not statistically significant. This was an unanticipated finding, most likely explained by two possibilities: 1 . due to technical difficulties, more time and effort was dedicated in scanning obese patients; 2 . due to limited sonographic windows (commonly 
observed in obese patient), improved consistency was obtained while measuring biometry. These hypotheses are theoretical since the study was not appropriately designed to test them ${ }^{18}$.

The strengths of the study are its prospective design, a well-defined protocol which allowed the inclusion of obese patients according to their BMI. Assessing the BMI in the third trimester at the time of the ultrasound exam (instead of pre-pregnancy BMI), allowed the real estimation of BMI's impact on weight estimation accuracy in clinical practice. Blinding was enforced during measurements to avoid adjusting the gestational age exhibited on the monitor, and in between exams to avoid sonographer's bias by seen each other's measurements. All measurements were obtained by standardized techniques with contemporary ultrasound equipment. Lastly, all ultrasound exams were performed by well trained, experienced sonographers and physicians ${ }^{18}$.

There are few limitations to this study. As mentioned in our original research, due to its pragmatic design to mimic common clinical conditions, the sample size might have been underpowered ${ }^{18}$. Additionally, it is unlikely that our results have external validity since the study was performed at a Maternal-Fetal medicine center with experienced sonographers and physicians. Lastly, the first author (JM) performed the study ultrasounds for a significant proportion of the patients while the rest of the ultrasounds were performed by multiple sonographers leading to an unknown effect of various sonographers on the results of statistical analysis. Since we found high accuracy of estimated fetal weight when compared to neonatal birthweight, there is a low concern for the possibility of inter-sonographer differences. Despite these limitations, our study is the first to systematically evaluate the effect of maternal obesity in the estimation of the fetal weight and has a very relevant clinical applicability in the context of the obesity epidemics.

In conclusion, when performed by trained sonographers, fetal sonographic weight estimation in the third trimester is accurate when compared to neonatal birthweight at increasing BMI categories.

\section{Declarations}

\section{Author's Contribution}

JG Martins: Project development, Data analysis, Data collection. Manuscript writing

T Kawakita: Data analysis, Manuscript review

P Jain: Data collection

M Gurganus: Data collection

D Baraki: Data collection

C Barake: Data collection, Manuscript writing 
E Sinkovskaya: Project development, Manuscript review

A Abuhamad: Project development, Data analysis, Manuscript review

\section{Statements and Declarations}

The authors declare that no funds, grants, or other support were received during the preparation of this manuscript.

The authors have no relevant financial or non-financial interests to disclose.

\section{References}

1. Sekar $R$, Khatun $M$, Barrett $H$, Duncombe $G$. A prospective pilot study in assessing the accuracy of ultrasound estimated fetal weight prior to delivery. Aust N Z J Obstet Gynaecol 2016; 56(1):49-53

2. Scioscia M, Vimercati A, Ceci O, Vicino M, Selvaggi L. Estimation of birth weight by two-dimensional ultrasonographu. Obstet Gynecol 2008; 111:57-65

3. Dudley N. A systematic review of the ultrasound estimation of fetal weight. Ultrasound Obstet Gynecol 2005; 25:80-89

4. Practice Bulletin No 156: Obesity in Pregnancy (Correction). Obstet Gynecol 2016; 128(6):1450

5. National Center for Health Statistics. Prevalence of obesity among adults and youth: United States 2017-2018. http://www.cdc.gov/nchs/products/databriefs/db360.htm. Accessed May 4, 2021

6. Kritzer S, Magner K, Warshak C. Increasing maternal body mass index and the accuracy of sonographic estimation of fetal weight near delivery. J Ultrasound Med 2014; 33:2173-2179

7. Racusin D, Stevens B, Campbell G, Aagard KM. Obesity and the risk and detection of fetal malformations. Semin Perinatol 2012; 36(3):213-21

8. Dashe JS, Mclntire DD, Twickler DM. Effects of maternal obesity on the ultrasound detection of anomalous fetuses. Obstet Gynecol 2009; 113:1001-1008

9. Dashe JS, Mclntire DD, Twickler DM. Maternal obesity limits the ultrasound evaluation of fetal anatomy. J Ultrasound Med 2009; 28:1025-1030

10. Aagaard-Tillery K, Flint Porter T, Malone F, Nyberg D, Collins J, Comstock C, Hankins G, Eddleman K, Dugoff L, Wolfe H, D'Alton M. Influence of maternal BMI on genetic sonography in the FaSTER trial. Prenat Diagn 2010;30(1):14-22

11. Policiano C, Mendes J, Fonseca A, Barros J, Martins D, Reis I, Clode N, Graca L. Impact of maternal weight on the intra-observer and inter-observer reproducibility of fetal ultrasound measurements in the third trimester. Int J Gynecol Obstet 2018;140:53-59

12. Heer IM, Kumper C, Vogtle N, Muller-Egloff S, Dugas M, Strauss A. Analysis of factor influencing the ultrasonic fetal estimation. Fetal Diagn Ther 2008; 23:204-210 
13. Farrell T, Holmes R, Stone P. The effect of body mass index on three methods of fetal weight estimation. BJOG 2002; 109:651-657

14. Cody F, Unterscheider J, Daly S, Geary MP, Malone FD et al. The effect of maternal obesity on sonographic fetal weight estimation and perinatal outcome in pregnancies complicated by fetal growth restriction. J Clin Ultrasound 2016; 44 (1):34-9

15. Aksoy H, Aksoy U, Karadag OI, Yucel B, Aydin T, Babayigit MA. Influence of maternal body mass index on sonographic fetal weight estimation prior to scheduled delivery. J Obstet Gynaecol Res $2015 ;$ 41:1556-1561

16. Paganelli S, Soncini E, Comitini G, Palomba S, La Sala GB. Sonogrpahic fetal weight estimation in normal and overweight / obese healthy term pregnant women by gestation-adjusted projection (GAP) method. Arch Gynecol Obstet 2016; 293(4):775-81

17. Blitz M, Rochelson B, Stork L, Augustine S, Greenberg M, Sison C, Vohra N. Effect of maternal body mass index on the accuracy of sonographic estimation of fetal weight in late gestation. Am $\mathrm{J}$ Perinatol 2018; 35:1235-1240

18. Martins JG, Kawakita T, Gurganus M, Baraki D, Jain P, Papageorghiou A, Abuhamad A. The influence of maternal body mass index on interobserver variability of fetal ultrasound biometry and amniotic fluid assessment in late pregnancy. Ultrasound Obstet Gynecol 2021, online ahead of print (PMID 33836119)

19. Abuhamad A et al. Obstetric and gynecology ultrasound curriculum and competency assessment in residency training programs: consensus report. Am J Obstet Gynecol 2018; 218 (1): 29-67

20. Hadlock FP, Harrist RB, Sharman RS, Deter RL, Park SK. Estimation of fetal weight with the use of head, body and femur measurements: a prospective study. Am J Obstet Gynecol 1985; 151: 333-337

21. Sarris I, loannou C et al. Intra- and interobserver variability in fetal ultrasound measurements. Ultrasound Obstet Gynecol 2012; 39: 266-273

22. Lima JC, Miyague AH, Filho FM, Nastri CO, Martins WP. Biometry and fetal weight estimation by twodimensional and three-dimensional ultrasonography: an intraobserver and interobserver reliability and agreement study. Ultrasound Obstet Gynecol 2012; 40:186-193

23. Little S, Edlow A, Thomas A, Smith N. Estimated fetal weight by ultrasound: a modifiable risk factor for cesarean delivery? Am J Obstet Gynecol 2012; 207 (4): 309.e1-6

24. Tsai PJ, Loichinger M, Zalud I. Obesity and the challenges of ultrasound fetal abnormality diagnosis. Best Pract Res Clin Obstet Gynaecol 2015;29:320-327

25. Stephens K, Al-Memar M, Lees C, et al. Comparing the relation between ultrasound-estimated fetal weight and birthweight in cohort of small-for-gestational-age fetuses. Acta Obstet Gynecol Scand 2019;98:1435-1441

26. Cavallaro A, Ash ST, Napolitano R et al. Quality control of ultrasound for fetal biometry: results from the INTERGROWTH-21st Project. Ultrasound Obstet Gynecol 2018; 52(3):332-339

\section{Tables}




\begin{tabular}{|c|c|c|c|c|c|c|}
\hline \multirow[b]{2}{*}{ Characteristic } & \multicolumn{6}{|c|}{ BMI Category } \\
\hline & $\begin{array}{c}\text { Total } \\
(n=100)\end{array}$ & $18-24.9(n=17)$ & $25.29 .9(n=27)$ & $30-39.9(n=29)$ & $240(n=27)$ & pvalue \\
\hline Gestational Age (weeks) at ultrasound & $36.8(35.6 ; 37.9)$ & $36.3(35.3 ; 37.9)$ & $36.9(35.6 ; 37.7)$ & $36.6(35.3 ; 37.6)$ & $37.1(36.1 ; 38.7)$ & 0.51 \\
\hline Maternal BMI $(\mathrm{kg} / \mathrm{m} 2)$ at ultrasound & $31.3(26.2 ; 41.2)$ & $23.6(22.4 ; 24.6)$ & $26.5(26.0 ; 27.5)$ & $34.4(31.6 ; 36.5)$ & $45.0(43.2 ; 47.7)$ & $<, 001$ \\
\hline Maternal Age (yr) & $25.9 \pm 5.3$ & $24.0 \pm 5.3$ & $25.7 \pm 5.7$ & $26.7 \pm 4.5$ & $26.6 \pm 5.6$ & 0.63 \\
\hline Gestational Age (weeks) at delivery & $39.4(38.9 ; 40.0)$ & $39.4(38.1 ; 40.1)$ & $39.4(38.7 ; 39.7)$ & $39.1(38.7 ; 40.0)$ & $39.6(39.1 ; 40.0)$ & 0.74 \\
\hline EFW by ultrasound (grams) & $2919(439)$ & $2774(382)$ & $2877(336)$ & $2862(479)$ & $3112(478)$ & 0.05 \\
\hline Birth weight (grams) & $3367(369)$ & $3230(416)$ & $3357(264)$ & $3329(369)$ & $3504(401)$ & 0.09 \\
\hline
\end{tabular}

Table 2. Z-scores, interobserver correlation coefficient and reliability coeffcients, for ultrasound estimated fetal weight and neonatal birthweight

\begin{tabular}{lllll}
\hline \multicolumn{1}{c}{ Fetal measurement } & Mean (SD) & Inter-CC (95\% CI) & Reliability coefficient & $\begin{array}{c}\text { Mean Z-score } \\
\text { difference (LOA) }\end{array}$ \\
\hline Z-score by ultrasound & $-0.17(0.81)$ & $0.69(0.57-0.78)$ & 0.82 & $0.08(-1.14$ to 1.31$)$ \\
\hline Z-score by birth weight & $-0.25(0.74)$ & & \multirow{2}{*}{$0.31)$} \\
\hline
\end{tabular}

Table 3.Z-scores, interobserver correlation coefficient and reliability coefficients by BMI category

\begin{tabular}{|c|c|c|c|c|}
\hline BMI Category & Inter-CC $(95 \% \mathrm{Cl})$ & Reliability coefficient & mean Z-score difference (LOA) & $p$ value \\
\hline $18-24.9(n=17)$ & $0.59(0.15-0.83)$ & 0.73 & $0.10(-1.30$ to 1.51$)$ & 0.92 \\
\hline $25-29.9(n=27)$ & $0.49(0.14-0.87)$ & 0.66 & $0.07(-1.12$ to 1.27$)$ & \\
\hline $30-39.9(n=29)$ & $0.73(0.50-0.87)$ & 0.84 & $0.03(-1.30$ to 1.35$)$ & \\
\hline$>=40(n=27)$ & $0.76(0.54-0.89)$ & 0.86 & $0.14(-0.94$ to 1.22$)$ & \\
\hline
\end{tabular}

\section{Figures}


Figure 1. Bland-Altman plot

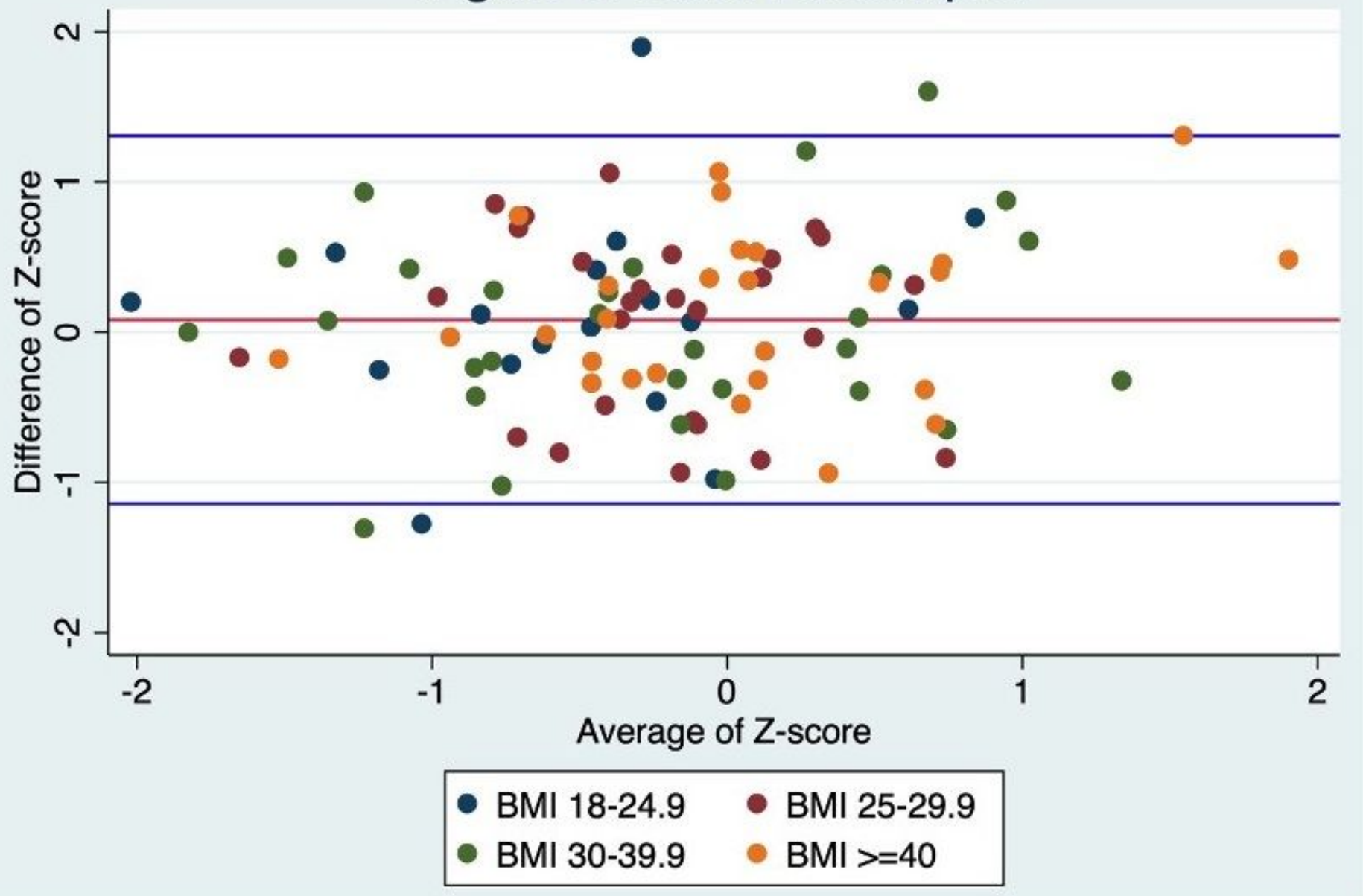

Figure 1

Bland-Altman plots for accuracy between sonographic estimated fetal weight and neonatal birthweight 